\title{
Prehospital evaluation of patients with chronic obstructive pulmonary disease
}

\author{
Mikkel Christensen*1, Jens Damgaard-Jensen ${ }^{2}$ and Henning Brinkenfeldt ${ }^{3}$
}

\author{
Address: ${ }^{1}$ Department of Internal Medicine, Amager Hospital, Denmark, ${ }^{2}$ Department of Anaesthesiology, Gentofte Hospital, Denmark and \\ ${ }^{3}$ Department of Anaesthesiology, Rigshospitalet, Denmark \\ Email: Mikkel Christensen* - drmikkelc@gmail.com \\ * Corresponding author
}

from Danish Society for Emergency Medicine: Research Symposium 2009

Copenhagen, Denmark. 26 April 2009

Published: 20 August 2009

Scandinavian Journal of Trauma, Resuscitation and Emergency Medicine 2009, I7(Suppl 2):PI0 doi:10.1 I86/I757-724I-17-S2-PI0

This abstract is available from: http://www.sjtrem.com/content/I7/S2/PIO

(C) 2009 Christensen et al; licensee BioMed Central Ltd.

\section{Introduction}

The mobile emergency care unit (MECU) in Copenhagen visits emergency patients in an urban setting and is manned by an experienced anesthesiologist and a specially trained fireman. The function of the MECU is to perform emergency treatment and evaluate the need for further treatment in a hospital. The aim of this study was to assess predictive measures of subsequent hospital admission in primarily completed patients with acute exacerbation of chronic obstructive pulmonary disease (COPD).

\section{Methods}

Through the MECU database we identified patients treated in the year 2002 with the primary diagnosis COPD. Depending on the MECU physician charts these patients were subdivided into a primary admitted group and a primary completed group. The MECU charts of the completed patients were then scrutinized for following parameters: age, sex, stethoscopic findings (normal or abnormal), saturation by pulse oxymetri (pre and post treatment), systolic blood pressure, treatment (inhaled beta-agonist and/or corticosteroid), and denial of hospital admission (yes or no). In addition, the completed patients were crosschecked with the Danish Central Personal Register for admissions within 72 hours of the MECU visit. Statistical analysis was performed comparing the primary treated patient with and without subsequent admission within 72 hours.

\section{Results}

In the year 2002 the MECU treated a total of 338 patients with COPD as primary diagnosis. Following initial treatment a large part, comprising $274(81 \%)$ of the COPD patients, were directly admitted to a hospital. 64 (19\%) of COPD patients were considered sufficiently treated by the emergency physician to stay in their own home. Of these patients left at home, $12(19 \%)$ were subsequently admitted in a hospital within $72 \mathrm{~h}$. The subsequent admitted patients were on average 7,5 years older than those who where not admitted $(\mathrm{p}<0.01)$. Regarding the other parameters there were no statistically significant differences between the two groups.

\section{Conclusion}

The evaluation of COPD patients with acute exacerbation is a considerable clinical challenge. The present study was not able to identify easy accessible clinical predictors, besides age, that in an acute setting allows discrimination between COPD patients, who needs admission within 72 hours and those who do not. 